\title{
Long-lasting olfactory dysfunction in COVID-19 patients
}

\author{
Bernhard Prem ${ }^{1}$ (D - David T. Liu ${ }^{1}$ - Gerold Besser ${ }^{1}$. Gunjan Sharma ${ }^{1} \cdot$ Laura E. Dultinger $^{1}$ - Sissy V. Hofer ${ }^{1}$. \\ Martina M. Matiasczyk ${ }^{1} \cdot$ Bertold Renner $^{2,3} \cdot$ Christian A. Mueller $^{1}$
}

Received: 29 July 2021 / Accepted: 20 October 2021 / Published online: 10 November 2021

(C) The Author(s) 2021

\begin{abstract}
Objectives Olfactory dysfunction (OD) is a common symptom of Coronavirus Disease 2019 (COVID-19). Although many patients have been reported to regain olfactory function within the first month, long-term observation reports vary. Therefore, we aimed to assess the course of chemosensory function in patients diagnosed with COVID-19 within 3-15 months after the infection.

Methods One hundred and two patients ( 71 females and 31 males; mean age 38.8 years) diagnosed with laboratory-confirmed COVID-19 and subjective OD participated in this single-center study 111-457 days after onset of OD. Patients first performed chemosensory tests at home, followed by psychophysical testing (Sniffin' Sticks (TDI), 27-item Candy Smell Test (CST), Taste Strips Test (TST)) in the clinic. Questionnaires regarding importance of olfaction (IOQ) and olfactory-specific quality of life (QOD) were applied at both timepoints.

Results After a mean 216 days (SD 73; range 111-457) between OD onset and follow-up testing, the mean Sniffin' Sticks (TDI) score was 27.1 points (SD 5.8; range 4.25-38.5): 4.0\% were anosmic, $72.5 \%$ hyposmic, and $23.5 \%$ normosmic. At follow-up testing, $73.5 \%$ of patients reported improvement, $5.9 \%$ deterioration, and $20.6 \%$ no change in OD. Moreover, full recovery of self-perceived smell, flavor, and taste was not observed. According to questionnaires, the individual importance of smell did not change, but participants showed improvement in OD-related quality of life $(p<0.001)$ and had increased parosmia scores $(p=0.014)$ at follow-up.

Conclusion Our results show that long-lasting OD after SARS-CoV-2 infection is a common symptom. The majority of patients had OD in the range of hyposmia, which was confirmed by comprehensive smell tests.
\end{abstract}

Keywords Anosmia $\cdot$ COVID-19 $\cdot$ Hyposmia $\cdot$ Olfaction $\cdot$ SARS-CoV-2 $\cdot$ Smell

\section{Introduction}

Since the first reported human infection with severe acute respiratory syndrome coronavirus-2 (SARS-CoV-2) in December 2019 in Wuhan, China, [1] coronavirus disease 2019 (COVID-19) has spread throughout the world [2]. The

Christian A. Mueller

christian.a.mueller@meduniwien.ac.at

1 Department of Otorhinolaryngology, Head and Neck Surgery, Medical University of Vienna, Vienna General Hospital, 1090 Vienna, Austria

2 Institute of Experimental and Clinical Pharmacology and Toxicology, Friedrich-Alexander Universität Erlangen-Nürnberg, Erlangen, Germany

3 Institute of Clinical Pharmacology, Medical Faculty Carl Gustav Carus, Technische Universität Dresden, Dresden, Germany course and symptoms of COVID-19 may differ considerably, ranging from mild almost asymptomatic disease to highly severe clinical progression, [3] with more than 3.1 million deceased by May 2021 [4]. Olfactory dysfunction (OD) and gustatory dysfunction are highly suspicious for COVID-19 and represent key symptoms in diagnosing infection with SARS-CoV-2 [5]. However, the prevalence of OD in COVID-19 patients differs due to the method of evaluation (anamnestic/subjective OD: 5\% [6] and 58.8\% [7] and psychophysical tests: 70\% [8] and 98\% [9]) or geographic region (54\% in Europe, 51\% in North America, 31\% in Asia, and $10 \%$ in Australia) [10].

Chemosensory dysfunction has primarily been reported based on subjective patient reports through surveys and questionnaires [6,7]. Subsequently, loss of chemosensory function has been assessed by psychophysical smell/taste tests. These protocols range from self-made home-based 
methods to validated, comprehensive chemosensory tests (e.g., the orthonasal olfactory Sniffin' Sticks test battery, threshold-discrimination-identification [TDI]) $[8,9]$. Reports on the duration of COVID-19-related OD have revealed that, 2 months after OD onset, $45 \%$ were diagnosed as hyposmic and $1 \%$ anosmic as measured by the TDI test [11]. Another study reported that, 5 weeks after symptom onset, 37\% had persistent smell loss [12]. However, observations of OD related to COVID-19 over a more extended period are still rare.

As data regarding this topic are urgently needed for patient counselling, we aimed to prospectively assess COVID-19-related smell loss using validated psychophysical tests and validated patient-reported outcome measures (PROMs) several months after the onset of OD.

\section{Materials and methods}

This study was approved by the ethics committee of the Medical University of Vienna (EK-No.: 1339/2020) and conducted according to the Declaration of Helsinki on biomedical research involving human subjects. All patients provided their written informed consent before participation.

\section{Patients}

In the present monocentric study, a total of 102 patients with COVID-19-related OD (71f/31 m; mean age: 38.8 years; standard deviation (SD): 13.2 years) were recruited by notices placed at the campus of the Medical University of Vienna and several newspapers. SARS-CoV-2 infection was proven either by polymerase chain reaction (PCR) during acute sickness $(n=74)$ or at the follow-up appointment at the Department of Otorhinolaryngology at the Medical University of Vienna based on blood samples positive for antibodies $\left(\right.$ Roche $\left.^{\odot}\right)$ against SARS-CoV-2 $(n=28)$. Patients $<18$ or $>85$ years of age or who had intolerance to sorbitol or fructose, head and neck tumours, dysphagia, head trauma, or neurological or sinonasal diseases were not eligible for the study. Furthermore, smell and taste loss before COVID-19 was considered as an exclusion criteria. According to the exclusion criteria, we had to rule out 19 interested subjects.

\section{Procedure}

The study was divided into two parts. First, PROMs, selfrating of smell/flavor/taste function, a retronasal screening test (the 7-item Candy Smell Test [7-CST]), and a screening method to evaluate taste function (short taste strips test [STST]) were performed at home. All necessary papers and tests were distributed by post. The results and further explanation of part I have been published elsewhere [13]. Thirteen patients, who have participated in part I, did not take part for testing in the clinic due to lack of interest.

The present study's investigations (part II) were carried out an average 216 days after the onset of OD. Two validated PROMs (the Questionnaire of Olfactory Disorders [QOD] and the Importance of Smell Questionnaire [IOQ]), selfassessment of smell/flavor/taste, the complete Sniffin' Sticks test battery (TDI), the 27-item Candy Smell Test (CST), and the full Taste Strips Test (TST) were performed. Examinations were carried out between August 2020 and May 2021 at the Department of Otorhinolaryngology at the Medical University of Vienna.

Upon inclusion, all participants were instructed to perform smell training with different odourants on a daily basis [14]. Olfactory training consists of repeated exposure to four different odourants twice a day and is safe as well as low cost. Although its underlying mechanism is still unknown, the benefit has been proven in multiple studies [14].

\section{PROMs and questionnaires}

The QOD assesses the olfactory-specific quality of life [15]. It consists of three parts evaluating negative statements (the extent to which patients suffer due to OD), positive statements (the degree to which patients cope with their OD), and parosmia score (the extent of qualitative OD symptomatology). Each answer is based on a 4-point Likert scale ranging from 0 (I agree) to 3 (I disagree). High results of negative statements (17 questions) are associated with a higher degree of suffering due to OD. Low results of positive statements (2 questions) suspect reduced capability of adjusting to OD. High scores on parosmia questions (4 questions) assume qualitative OD [16].

The IOQ consists of 20 questions related to the individual importance of smell $[17,18]$. The patients must pick one of four answers ranging from 0 (I totally disagree) to 3 (I totally agree). Higher individual significance of olfaction is associated with a higher score.

\section{Self-assessment of smell, flavor, and taste function}

Participants classified their self-assessed chemosensory functions of smell (SAS), flavor (SAF), and taste (SAT) before the psychophysical tests. In addition, participants rated their chemosensory functions during home testing before infection (retrospectively) and on the day of home testing. Numeric rating scales ranged from 1 (very bad) to 10 (very good). All participants were informed about chemosensory functions to differentiate smell, flavor and taste. 


\section{Chemosensory testing}

Orthonasal olfactory function was measured by the comprehensive Sniffin' Sticks test battery (TDI, Burghart Medical Technology, Wedel, Germany) [19]. This consists of three subtests: odor threshold (T), odor discrimination (D), and odor identification (I). Each subtest can achieve 16 points, resulting in a maximum score of 48 . This test is based on a forced-choice paradigm and allows differentiation between normosmia, hyposmia, and functional anosmia. According to normative data, scores $\leq 16$ represent anosmia, $>16$ and $<31$ represent hyposmia, and from 31 to 48 normosmia [20].The exact testing procedure is described elsewhere $[19,21]$.

Retronasal olfactory function was assessed by the 27 -item version [22] of the CST [23]. Each flavored candy consists of $500 \mathrm{mg}$ sorbitol and a unique aroma. Candies are placed on the tongue, and the participant must pick one of four answers following the forced-choice paradigm. After each candy, the participant takes a sip of water or rinses their mouth with water. Studies have proven the general applicability of selfadministration [22,24] and postal distribution [22].

By applying the TST, we sought to evaluate gustatory function [25]. Filter paper strips impregnated with four taste solutions (sweet, sour, salty, bitter) at four concentrations, as well as two blanks, formed the complete TST set. Patients had to choose one of five answers (sweet, sour, salty, bitter, or no taste). After each taste strip, the participants took a sip of water or rinsed their mouth with water. According to normative data, [25] scores between 9 and 16 represent normgeusia and scores between 1 and 8 hypogeusia. Thus, 0 points denotes ageusia. Detailed descriptions of the TST are given elsewhere [25].

\section{Statistical analysis}

IBM SPSS 26.0 (IBM Corp., Armonk, NY, USA) and Graph-Prism 8.4.3 (GraphPad Software, Inc., La Jolla, CA, USA) were used for statistical analyses and graphical visualization. Utilizing histograms, we verified the normality of data distributions. Group comparisons were analyzed based on Student's $t$ test. For multiple group comparisons, we used one-way repeated-measures analysis of variance (rm-ANOVA), followed by Tukey's post-hoc test. We utilized Pearson's correlation coefficient ( $r$ ) for bivariate correlations, where we interpreted $r>0.7$ as strong, 0.4-0.7 as moderate, and $<0.4$ as weak. $P<0.05$ was considered significant.

\section{Results}

Thirty-one men and 71 women with a mean age of 38.8 years (SD 13.2; range 18-68) performed home and follow-up testing of chemosensory function. The mean duration between the onset of OD and home testing was 57 days (SD 50). Between home testing and follow-up testing in the present investigation, another 159 days (SD 55) passed. Thus, 216 days (SD 74) elapsed from OD onset until follow-up testing (Table 1).

\section{Follow-up chemosensory testing}

Orthonasal psychophysical testing using the TDI had a mean score of 27.1 points (SD 5.8; range 4.25-38.5). Compared to recently updated normative cutoff data, $23.5 \%$ were classified as normosmic, $72.5 \%$ hyposmic, and $4.0 \%$ anosmic (Table 2). Evaluation of retronasal olfactory function by the CST resulted in a mean score of 16.8 (SD 5.0; range 4-24). Assessment of taste function by the TST yielded a mean score of 11.0 (SD 2.6; range 3-15). According to normative cutoff data, normgeusia was found in $81.4 \%$ and hypogeusia in $18.6 \%$ of patients (Table 2).

Furthermore, no correlation was found between the chemosensory test results (TDI, CST, and TST) and the duration between onset of OD and follow-up testing ( $p=0.116$, $p=0.390, p=0.543$, respectively).

To evaluate differences in the dependence of OD duration, we separated the study cohort into two groups. Group A consisted of participants who implemented home testing within 30 days after the onset of OD. Therefore, patients who completed home testing $\geq 31$ days after the start of OD formed Group B. Comparing the results of the two groups did not reveal significant differences regarding the chemosensory tests (TDI: $p=0.827$; CST: $p=0.282$; TST: $p=0.430$ ) (Table 2).

\section{Correlations between self-reported and psychophysically evaluated chemosensory functions}

Individual self-assessment of smell and TDI results at follow-up revealed a moderate correlation $\left(r_{102}=0.429\right.$; $p<0.001)$. Furthermore, weak but significant correspondence between SAT and TST was found $\left(r_{102}=0.258\right.$; $p=0.009)$. Moreover, analyzing SAF and CST showed a moderate correlation $\left(r_{102}=0.551 ; p<0.001\right)$.

\section{Course of self-perceived chemosensory function}

We also compared the self-assessment of chemosensory function over the course of time (Fig. 1). 
Table 1 Descriptive statistics $(N=102)$

Descriptive statistics

\section{Gender}

Age, years

Duration between onset of OD and home testing, days

Duration between home and follow-up testing, days

Duration between onset of OD and follow-up testing, days

Proof of COVID-19 infection via

Self-Assessment

Smell

Flavor

Taste

Questionnaires

Female: 71/Male: 31

Mean 38.8 (SD 13.2); range 18-68

Mean 57 (SD 50); range 7-374

Mean 159 (SD 55); range 83-301

Mean 216 (SD 74); range 111-457

PCR: 74

AB: 28

Before OD/Home testing/Followup [Mean (SD)]

$9.3(1.2) / 4.1(2.7) / 6.0(2.8)$

$9.2(1.2) / 5.0(2.8) / 6.1(2.9)$

$9.3(1.4) / 6.0(2.6) / 7.3(2.2)$

Home testing/Follow-up [Mean (SD)]

\section{IOQ}

QOD-Parosmia

QOD-NS

QOD-PS

Psychophysical tests: home testing

7-CST

STST

CST 27-item Candy Smell Test; 7-CST 7-item Candy Smell Test; $A B$ Antibodies; IOQ Importance of Olfaction Questionnaire; $O D$ olfactory dysfunction; PCR polymerase-chain-reaction; $Q O D$-NS Questionnaire of Olfactory Disorders-Negative Statements; QOD-Parosmia Questionnaire of Olfactory Disorders-Parosmia Score; QOD-PS Questionnaire of Olfactory Disorders-Positive Statements; SD standard deviation; STST Suprathreshold Taste Strips Test; TDI threshold discrimination identification (Sniffin' Sticks); TST Taste Strips Test

Table 2 Results of follow-up psychophysical tests

\begin{tabular}{llllll}
\hline & TDI & Distribution according to TDI* & CST & TST & Distribution according to TST $^{\#}$ \\
\hline All participants $(n=102)$ & $27.1(5.8)$ & $\begin{array}{l}\text { Anosmic: } 4.0 \% \\
\text { Hyposmic: } 72.5 \% \\
\text { Normosmic: } 23.5 \%\end{array}$ & $16.8(5.0)$ & $11.0(2.6)$ & $\begin{array}{l}\text { Hypogeusia: } 18.6 \% \\
\text { Normgeusia: } 81.4 \%\end{array}$ \\
Group A $(n=32)$ & $27.3^{\mathrm{ns}}(6.6)$ & $\begin{array}{l}\text { Anosmic: } 6.3 \% \\
\text { Hyposmic: } 65.6 \%\end{array}$ & $17.6^{\mathrm{ns}}(5.4)$ & $11.2^{\mathrm{ns}}(2.5)$ & $\begin{array}{l}\text { Hypogeusia: } 18.8 \% \\
\text { Normgeusia: } 81.3 \%\end{array}$ \\
& \multirow{2}{*}{$\begin{array}{l}\text { Normosmic: } 28.1 \% \\
\text { Group B }(n=70)\end{array}$} & $\begin{array}{l}\text { Anosmic: } 4.3 \% \\
\text { Hyposmic: } 74.3 \% \\
\text { Normosmic: } 21.4 \%\end{array}$ & $16.4^{\mathrm{ns}}(4.9)$ & $10.8^{\mathrm{ns}}(2.7)$ & Hypogeusia: $18.6 \%$ \\
& & & & Normgeusia: $81.4 \%$ \\
\hline
\end{tabular}

Data are given as mean (SD) unless otherwise noted

Group A: implemented home testing within 30 days after the onset of olfactory dysfunction (OD); Group B: performed home testing beyond 30 days after the onset of $\mathrm{OD}$

CST 27-item Candy Smell Test; SD standard deviation; TDI threshold discrimination identification (Sniffin' Sticks); TST Taste Strips Test

*According to Oleskiewicz et al. [20]

\#According to Mueller et al. [25]

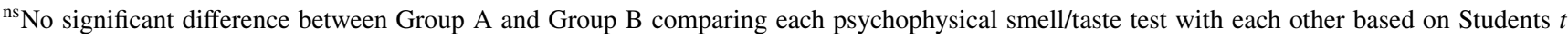
test 

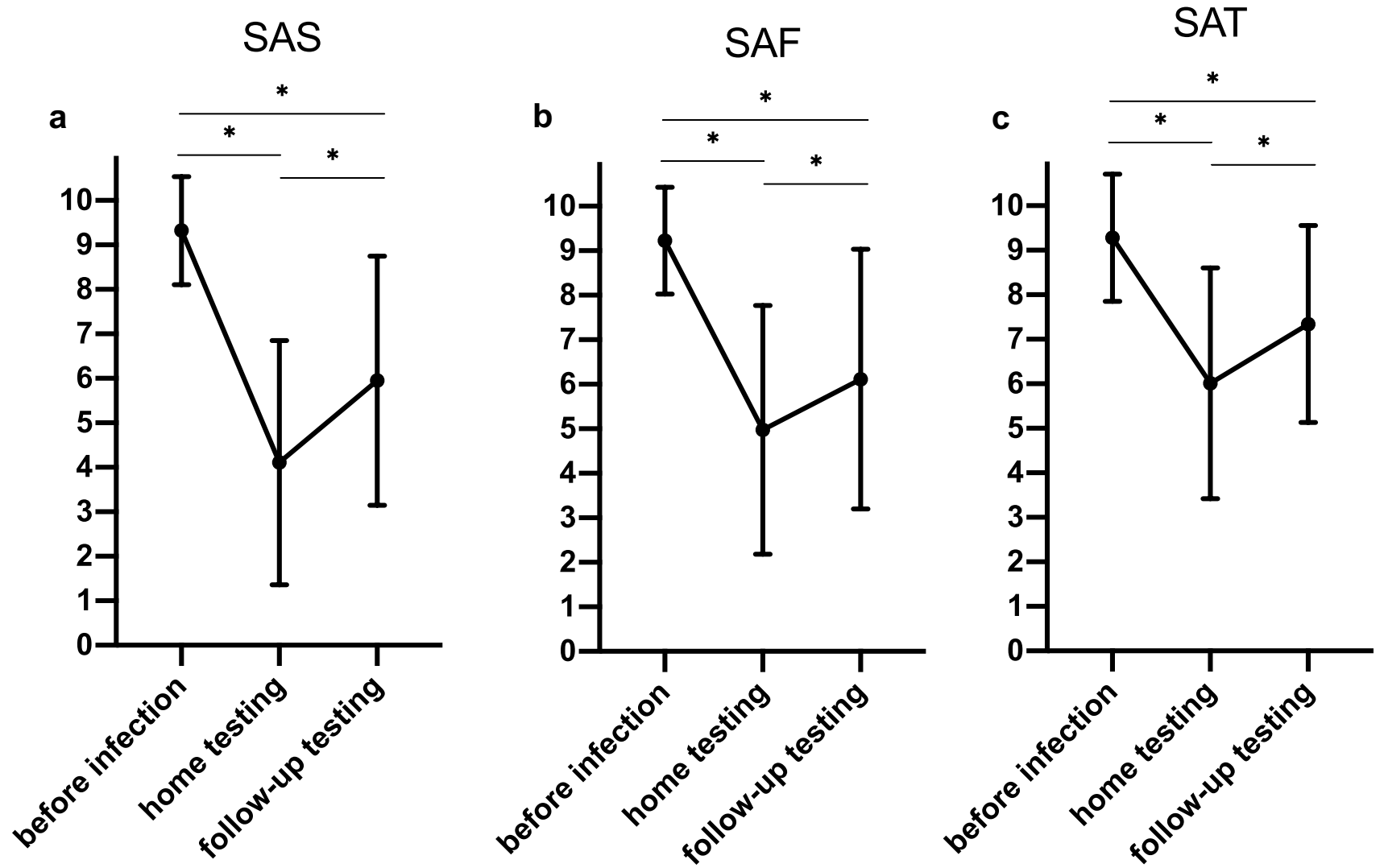

Fig. 1 Course of self-reported chemosensory functions. Scale: 1 (very bad) to 10 (very good). *significant difference based on oneway repeated-measures ANOVA, followed by Tukey's post-hoc test.

$S A S$ self-assessment of smell; $S A F$ self-assessment of flavor; $S A T$ self-assessment of taste

One-way rm-ANOVA for SAS, SAF, and SAT showed significant differences across the observational period $(F(101$, $202)=2.243, p<0.001 ; F(101,202)=2.138, p<0.001$; and $F(101,202)=1.845, p<0.001$, respectively). Tukey's posthoc test revealed significant deterioration of all qualities (smell, flavor, and taste) within the acute phase of SARSCoV-2 infection. Furthermore, Tukey's post-hoc test showed significant improvement in all chemosensory qualities when evaluated during follow-up testing. Nevertheless, analyzing the self-assessment of these qualities before OD and during the follow-up appointment, Tukey's post-hoc test still revealed a significant difference.

\section{Course of the IOQ}

To evaluate the importance of olfaction over time, we applied the IOQ during home and follow-up testing (Table 1). The comparison (paired $t$ test) of the individual IOQ scores revealed no significant difference.

\section{Course of daily life problems due to OD}

We applied the QOD to evaluate the daily life problems caused by OD and whether patients adapt over time (Table 1). The positive statement score (the capability of adjusting to OD) and the score for negative statements (the extent to which patients suffer from OD) decreased significantly ( $p=0.029 ; p<0.001$, respectively). However, the parosmia score increased significantly between home and follow-up testing $(p=0.014)$.

\section{Discussion}

Investigating olfactory dysfunction in COVID-19 patients over a long period of time, we had three main findings. First, measuring olfactory function revealed hyposmia in the majority of investigated patients up to 15 months after symptom onset. Second, self-assessed smell function improved significantly compared to initial loss, but did not reach the level of function before the disease. Third, according to questionnaires, the importance of smell did not change with 
time, but parosmia increased and the extent of suffering due to OD decreased significantly.

Few studies have assessed olfactory function in patients with SARS-CoV-2-related smell loss using PROMs and psychophysical tests [12,26-28]. Solely asking for smell function frequently leads to incorrect results [29-31]. Therefore, it is important to combine PROMs and tests of chemosensory function to uncover the course of the regeneration of smell loss [14].

Among our patients, only $23.5 \%$ achieved normosmia after a mean duration of smell loss of 216 days, with the vast majority $(76.5 \%)$ still exhibiting some degree of OD. Our study provides further evidence that a considerable group of patients with SARS-CoV-2-related smell loss does not recover rapidly, which is in contrast to the pandemic's initial reports [12, 26, 28]. Most reports early in the pandemic suggested that most patients regain normal smell function after a short period of time. Reiter et al. reported that $72 \%$ recovered from OD within 1 month based on anamnestic evaluation [32]. Other colleagues reported similar results: $63 \%$ within 1-4 weeks, [26] $62 \%$ within 5 weeks, [12] and $75 \%$ within 2 months [28]. Few long-term observations exist: Niklaasen et al. reported that most of the affected people recovered within 28 days, but approximately $27 \%$ still suffered from different degrees of OD by applying TDI 28-169 days after the onset of OD [27]. Another multicenter study reported that approximately $95 \%$ regained their ability to smell after 6 months by implementing psychophysical smell tests [33]. By applying the culturally adapted University of Pennsylvania Smell Identification Test, Boscolo-Rizzo et al. found that $60 \%$ still suffered from different degrees of OD 6 months after the onset of OD [34]. A 6-month online follow-up survey showed that approximately $36 \%$ of patients affected by COVID-19-associated OD complained about persistent reduced olfactory function [35].

Concerning the degree of persistent OD, we found that most patients scored within the range of hyposmia. Only $4 \%$ of the investigated patients were diagnosed with functional anosmia 3-15 months after disease onset. This leads to the assumption that OD due to infection with SARS-CoV-2, though lasting a long time in some patients, may not permanently disrupt the olfactory epithelium in most cases and leave a subgroup of olfactory neurons intact. This could be because specific ACE receptors responsible for the invasion of SARS-CoV-2 are primarily located on olfactory sustentacular cells, [36,37] leaving olfactory receptor neurons less affected.

Although we cannot report the exact percentage of patients with persistent smell loss (longer than 4 weeks), it seems obvious that a notable proportion of infected patients do not recover as quickly as initially reported. Potential support for this theory is that we did not find differences between patients included within the first 30 days of OD and those included later. Consequently, patients with rapid recovery of smell function who experienced OD only for a brief period of time (maximum 2 weeks) may not have participated in the present study. The results of the self-assessment of smell, taste, and flavor in our patients may support these considerations, because the majority of self-perceived functions failed to recover fully. During acute infection and home testing, the self-ratings of chemosensory functions decreased significantly. However, at follow-up testing, they increased significantly but still differed significantly from the pre-OD values. However, in this case our study has one limitation: patients rated their chemosensory functions before infection retrospectively, at the same time when they evaluated the functions of smell, flavor, and taste during home testing. Therefore, it may be possible that patients rated their chemosensory abilities retrospectively too well. Furthermore, consistent with previous studies, our results revealed only weak to moderate correlations between self-assessed and psychophysical evaluations of chemosensory functions [38].

Moreover, recruitment of these studies' patients' needs to be discussed as potential selection bias. In our study, patients with proven SARS-CoV-2 infection and subjective OD contacted us for inclusion. Thus, mainly olfactory highly attentive and interested patients, who suffer severely from smell malfunction may be included into this study. Furthermore, the gender distribution may allow conclusion that more women than men are interested into individual olfactory function and improvement of its dysfunction.

Regarding the olfactory-related quality of life, the negative statement score of the QOD decreased significantly, meaning less suffering from OD. Furthermore, the parosmia score increased significantly, indicating the emergence of qualitative OD due to SARS-CoV-2 infection over time. The individual importance of olfaction did not change within 3-15 months. This may indicate that, even after several months of decreased olfactory function, patients desired recovery of their chemosensory function and consequently seemed highly interested in adequate treatment.

According to the current Position Paper on Olfactory Dysfunction [14], olfactory training is the recommended therapy for post-infectious OD. Based on multiple studies that reveal beneficial features of olfactory training [39-42], daily practicing with four odourants has become standard recommendation of COVID-19 related OD. Despite smell training, different centers studied the effect of cortisoneintranasal as well as oral - on the outcome regarding OD. In a case-control study with 50 patients in each group (olfactory training solely vs. olfactory training and intranasal cortisone) the self-assessment of smell did not differ between the groups [41]. Furthermore, Kasiri et al. revealed no significant difference concerning SAS and psychophysical tests (the Iran Smell Identification Test) between intervention (intranasal cortisone and olfactory training) and control 
(solely olfactory training) group [42]. Whereas, another case-control study showed a significant improvement of psychophysically evaluated smell function after olfactory training and 10 days of oral cortisone. The results of the control group (only smell training) did not change significantly [40]. Nevertheless, besides the proven effectiveness of olfactory training, the value of cortisone needs to be evaluated in larger studies.

As the pandemic is still ongoing in most affected countries and vaccination of the majority of the population will last months, to even years, knowledge and recognition of the course of the disease is of great importance regarding patient management. Therefore, as long as therapy for postinfectious smell loss is restricted to smell training, patients need to be counseled appropriately. Currently, based on our results, SARS-CoV-2-related smell loss seems to last longer in select patients than previously thought, with the majority of patients in the hyposmic range. Anosmia, the complete loss of the sense of smell, seems to be rare. Consequently, smell training specifically should be advocated, as the degree of OD constitutes a prognostic factor regarding the success of olfactory training [43]. Nevertheless, further research is urgently needed regarding factors that influence prolonged COVID-19-related smell loss.

\section{Conclusion}

Olfactory dysfunction is a common and well-known symptom of COVID-19. Although previous investigations have shown that most patients recover within the first few weeks, our results highlight that knowledge about long-lasting OD related to SARS-CoV-2 infection is an essential aspect of patient management.

Acknowledgements We also thank S. Seyferth for the manufactured candies and Frey\&Lau GmbH for providing the aromas.

Funding Open access funding provided by Medical University of Vienna. We thank the mayor of the city of Vienna for supporting the project with "Stiftungsfonds zur Foerderung der Bekaempfung der Tuberkulose und anderer Lungenkrankheiten".

\section{Declarations}

Conflict of interest All authors declare no conflicts of interest regarding this paper.

Open Access This article is licensed under a Creative Commons Attribution 4.0 International License, which permits use, sharing, adaptation, distribution and reproduction in any medium or format, as long as you give appropriate credit to the original author(s) and the source, provide a link to the Creative Commons licence, and indicate if changes were made. The images or other third party material in this article are included in the article's Creative Commons licence, unless indicated otherwise in a credit line to the material. If material is not included in the article's Creative Commons licence and your intended use is not permitted by statutory regulation or exceeds the permitted use, you will need to obtain permission directly from the copyright holder. To view a copy of this licence, visit http://creativecommons.org/licenses/by/4.0/.

\section{References}

1. Wang C, Horby PW, Hayden FG, Gao GF (2020) A novel coronavirus outbreak of global health concern. Lancet 395(10223):470 473. https://doi.org/10.1016/s0140-6736(20)30185-9

2. WHO Director-General's opening remarks at the media briefing on COVID-19 - March 11 2020. 11th of March 2020, https://www. who.int/director-general/speeches/detail/who-director-general-sopening-remarks-at-the-media-briefing-on-covid-19. Accessed 11 Mar 2020

3. Pascarella G, Strumia A, Piliego C et al (2020) COVID-19 diagnosis and management: a comprehensive review. J Intern Med 288(2):192-206. https://doi.org/10.1111/joim.13091

4. Organization WH. COVID-19 Weekly Epidemiological Update. 2021; https://www.who.int/publications/m/item/weekly-epide miological-update-on-covid-19 Accessed 4 May 2021

5. Hopkins C, Surda P, Kumar N (2020) Presentation of new onset anosmia during the COVID-19 pandemic. Rhinology. https://doi. org/10.4193/Rhin20.116

6. Mao L, Jin H, Wang M, Hu Y, Chen S, He Q, Chang J, Hong C, Zhou Y, Wang D, Miao X, Li Y, Hu B (2020) Neurologic manifestations of hospitalized patients With coronavirus disease 2019 in Wuhan, China. JAMA Neurol 77(6):683-690. https://doi.org/ 10.1001/jamaneurol.2020.1127

7. Abalo-Lojo JM, Pouso-Diz JM, Gonzalez F (2020) Taste and smell dysfunction in COVID-19 patients. Ann Otol Rhinol Laryngol. https://doi.org/10.1177/0003489420932617

8. Lechien JR, Cabaraux P, Chiesa-Estomba CM et al (2020) Psychophysical olfactory tests and detection of COVID-19 in Patients with sudden onset olfactory dysfunction: a prospective study. Ear Nose Throat J. https://doi.org/10.1177/0145561320 929169

9. Moein ST, Hashemian SMR, Mansourafshar B, Khorram-Tousi A, Tabarsi P, Doty RL (2020) Smell dysfunction: a biomarker for COVID-19. Int Forum Allergy Rhinol. https://doi.org/10.1002/ alr.22587

10. Saniasiaya J, Islam MA, Abdullah B (2020) Prevalence of olfactory dysfunction in coronavirus disease 2019 (COVID-19): a meta-analysis of 27,492 patients. Laryngoscope. https://doi.org/ 10.1002/lary.29286

11. Otte MS, Eckel HNC, Poluschkin L, Klussmann JP, Luers JC (2020) Olfactory dysfunction in patients after recovering from COVID-19. Acta Otolaryngol. https://doi.org/10.1080/00016489. 2020.1811999

12. Le Bon SD, Pisarski N, Verbeke J et al (2020) Psychophysical evaluation of chemosensory functions 5 weeks after olfactory loss due to COVID-19: a prospective cohort study on 72 patients. Eur Arch Otorhinolaryngol. https://doi.org/10.1007/ s00405-020-06267-2

13. Prem B, Liu DT, Besser G, Renner B, Mueller CA (2021) Retronasal olfactory testing in early diagnosed and suspected COVID-19 patients: a 7-week follow-up study. Eur Arch Otorhinolaryngol. https://doi.org/10.1007/s00405-021-06826-1

14. Hummel T, Whitcroft KL, Andrews P et al (2017) Position paper on olfactory dysfunction. Rhinol Suppl 54(26):1-30. https://doi. org/10.4193/Rhino16.248 
15. Frasnelli J, Hummel T (2005) Olfactory dysfunction and daily life. Eur Arch Otorhinolaryngol 262(3):231-235. https://doi.org/ 10.1007/s00405-004-0796-y

16. Liu DT, Besser G, Prem B et al (2020) Self-perceived taste and flavor perception: associations with quality of life in patients with olfactory loss. Otolaryngol Head Neck Surg. https://doi.org/10. 1177/0194599820965242

17. Croy I, Buschhuter D, Seo HS, Negoias S, Hummel T (2010) Individual significance of olfaction: development of a questionnaire. Eur Arch Otorhinolaryngol 267(1):67-71. https://doi.org/ 10.1007/s00405-009-1054-0

18. Croy I, Landis BN, Meusel T, Seo HS, Krone F, Hummel T (2011) Patient adjustment to reduced olfactory function. Arch Otolaryngol Head Neck Surg 137(4):377-382. https://doi.org/10.1001/ archoto.2011.32

19. Kobal G, Hummel T, Sekinger B, Barz S, Roscher S, Wolf S (1996) "Sniffin' sticks": screening of olfactory performance. Rhinology 34(4):222-226

20. Oleszkiewicz A, Schriever VA, Croy I, Haehner A, Hummel T (2019) Updated Sniffin' Sticks normative data based on an extended sample of 9139 subjects. Eur Arch Otorhinolaryngol 276(3):719-728. https://doi.org/10.1007/s00405-018-5248-1

21. Hummel T, Kobal G, Gudziol H, Mackay-Sim A (2006) Normative data for the "Sniffin" Sticks" including tests of odor identification, odor discrimination, and olfactory thresholds: an upgrade based on a group of more than 3,000 subjects." Eur Arch Otorhinolaryngol 264(3):237-243. https://doi.org/10.1007/ s00405-006-0173-0

22. Besser G, Tianxiang Liu D, Prem B et al (2020) Retronasal olfactory testing using candies sent by post and for screening purposes: a feasibility study. Rhinology 58(3):218-225. https://doi.org/10. 4193/Rhin19.230

23. Renner B, Mueller CA, Dreier J, Faulhaber S, Rascher W, Kobal G (2009) The candy smell test: a new test for retronasal olfactory performance. Laryngoscope 119(3):487-495. https://doi.org/10. 1002/lary.20123

24. Haxel BR, Bertz-Duffy S, Faldum A et al (2011) The Candy Smell Test in clinical routine. Am J Rhinol Allergy 25(4):e145-e148. https://doi.org/10.2500/ajra.2011.25.3611

25. Mueller CA, Kallert S, Renner B et al (2003) Quantitative assessment of gustatory function in a clinical context using impregnated "taste strips." Rhinology 41(1):2-6

26. Moein ST, Hashemian SM, Tabarsi P, Doty RL (2020) Prevalence and reversibility of smell dysfunction measured psychophysically in a cohort of COVID-19 patients. Int Forum Allergy Rhinol. https://doi.org/10.1002/alr.22680

27. Niklassen AS, Draf J, Huart C et al (2021) COVID-19: recovery from chemosensory dysfunction. A multicentre study on smell and taste. Laryngoscope. https://doi.org/10.1002/lary.29383

28. Lechien JR, Chiesa-Estomba CM, Beckers E, Mustin V, Ducarme M, Journe F, Marchant A, Jouffe L, Barillari MR, Cammaroto G, Circiu MP, Hans S, Saussez S (2021) Prevalence and 6-month recovery of olfactory dysfunction: a multicentre study of 1363 COVID-19 patients. J Intern Med 290(2):451-461. https://doi. org/10.1111/joim.13209

29. Landis BN, Hummel T, Hugentobler M, Giger R, Lacroix JS (2003) Ratings of overall olfactory function. Chem Senses 28(8):691-694. https://doi.org/10.1093/chemse/bjg061

30. Welge-Luessen A, Hummel T, Stojan T, Wolfensberger M (2018) What is the correlation between ratings and measures of olfactory function in patients with olfactory loss? Am J Rhinol 19(6):567571. https://doi.org/10.1177/194589240501900606

31. Lotsch J, Hummel T (2019) Clinical usefulness of self-rated olfactory performance-a data science-based assessment of 6000 patients. Chem Senses 44(6):357-364. https://doi.org/10.1093/ chemse/bjz029

32. Reiter ER, Coelho DH, Kons ZA, Costanzo RM (2020) Subjective smell and taste changes during the COVID-19 pandemic: short term recovery. Am J Otolaryngol 41(6):102639. https://doi.org/ 10.1016/j.amjoto.2020.102639

33. Lechien JR, Chiesa-Estomba CM, Beckers E et al (2021) Prevalence and 6-month recovery of olfactory dysfunction: a multicentre study of 1363 COVID-19 patients. J Intern Med. https://doi. org/10.1111/joim.13209

34. Boscolo-Rizzo P, Hummel T, Hopkins C, Dibattista M, Menini A, Spinato G, Fabbris C, Emanuelli E, D'Alessandro A, Marzolino R, Zanelli E, Cancellieri E, Cargnelutti K, Fadda S, Borsetto D, Vaira LA, Gardenal N, Polesel J, Tirelli G (2021) High prevalence of long-term olfactory, gustatory, and chemesthesis dysfunction in post-COVID-19 patients: a matched case-control study with oneyear follow-up using a comprehensive psychophysical evaluation. Rhinology. https://doi.org/10.4193/Rhin21.249

35. Lucidi D, Molinari G, Silvestri M et al (2021) Patient-reported olfactory recovery after SARS-CoV-2 infection: a 6-month followup study. Int Forum Allergy Rhinol. https://doi.org/10.1002/alr. 22775

36. Bilinska K, Jakubowska P, Von Bartheld CS, Butowt R (2020) Expression of the SARS-CoV-2 entry proteins, ACE2 and TMPRSS2, in cells of the olfactory epithelium: identification of cell types and trends with age. ACS Chem Neurosci. https://doi. org/10.1021/acschemneuro.0c00210

37. Brann DH, Tsukahara T, Weinreb C et al (2020) Non-neuronal expression of SARS-CoV-2 entry genes in the olfactory system suggests mechanisms underlying COVID-19-associated anosmia. Sci Adv. https://doi.org/10.1126/sciadv.abc5801

38. Liu DT, Besser G, Prem B et al (2020) Association between orthonasal olfaction and chemosensory perception in patients with smell loss. Laryngoscope. https://doi.org/10.1002/lary.28773

39. Denis F, Septans AL, Periers L et al (2021) Olfactory training and visual stimulation assisted by a web application for patients with persistent olfactory dysfunction after SARS-CoV-2 infection: observational study. J Med Internet Res 23(5):e29583. https://doi. org/10.2196/29583

40. Le Bon SD, Konopnicki D, Pisarski N, Prunier L, Lechien JR, Horoi M (2021) Efficacy and safety of oral corticosteroids and olfactory training in the management of COVID-19-related loss of smell. Eur Arch Otorhinolaryngol. https://doi.org/10.1007/ s00405-020-06520-8

41. Abdelalim AA, Mohamady AA, Elsayed RA, Elawady MA, Ghallab AF (2021) Corticosteroid nasal spray for recovery of smell sensation in COVID-19 patients: a randomized controlled trial. Am J Otolaryngol 42(2):102884. https://doi.org/10.1016/j. amjoto.2020.102884

42. Kasiri H, Rouhani N, Salehifar E, Ghazaeian M, Fallah S (2021) Mometasone furoate nasal spray in the treatment of patients with COVID-19 olfactory dysfunction: a randomized, double blind clinical trial. Int Immunopharmacol 98:107871. https://doi.org/ 10.1016/j.intimp.2021.107871

43. Liu DT, Pellegrino R, Sabha M et al (2021) Factors associated with relevant olfactory recovery after olfactory training: a retrospective study including 601 participants. Rhinology 59:91-97. https://doi.org/10.4193/Rhin20-262

Publisher's Note Springer Nature remains neutral with regard to jurisdictional claims in published maps and institutional affiliations. 